\title{
Explicit Model Predictive Control and L1-Navigation Strategies for Fixed-Wing UAV Path Tracking
}

\section{Conference Paper}

\section{Author(s):}

Oettershagen, Philipp; Melzer, Amir; Leutenegger, Stefan; Alexis, Kostas; Siegwart, Roland

Publication date:

2014

Permanent link:

https://doi.org/10.3929/ethz-a-010589813

Rights / license:

In Copyright - Non-Commercial Use Permitted

Originally published in:

https://doi.org/10.1109/MED.2014.6961532 


\title{
Explicit Model Predictive Control and $\mathcal{L}_{1}$-Navigation Strategies for Fixed-Wing UAV Path Tracking
}

\author{
Philipp Oettershagen, Amir Melzer, Stefan Leutenegger, Kostas Alexis and Roland Siegwart
}

\begin{abstract}
A control strategy for fixed-wing Unmanned Aerial Vehicles is proposed and relies on the combination of linear model predictive control laws for the attitude dynamics of the system, along with an implementation of the $\mathcal{L}_{1}$-navigation logic that provides attitude reference commands to achieve precise path tracking. The employed predictive controllers ensure the performance characteristics of the critical attitude loops, while respecting the actuation limitations of the platform along with safety considerations encoded as state constraints. Being explicitly computed, these strategies are computationally lightweight and allow for seamless integration on the onboard avionics. Once the desired attitude response characteristics are achieved, tuning the cascaded nonlinear $\mathcal{L}_{1}$-navigation law becomes straightforward as lateral acceleration references can be precisely tracked. A wide set of experiments was conducted in order to evaluate the performance of the proposed strategies. As shown high quality tracking results are achieved.
\end{abstract}

\section{INTRODUCTION}

Unmanned Aerial Vehicles (UAVs) have already proven their potential on a large set of civilian operations such as search and rescue [1], crop monitoring [2], infrastructure inspection [3], mapping [4] and more. Among all possible types of UAVs, fixed-wing systems pose the fundamental advantage of prolonged endurance and increased payloads which make them the most appropriate choice for a large subset of critical real-life applications. However, the need for simple to deploy, easy to operate and user-friendly systems leads to miniaturization which consequently has a significant impact on the inherent robustness of the platforms, the capabilities of the onboard sensorial and processing modules and the actuator properties. Therefore, a new family of control laws with performance and safety guarantees should be developed in order to ensure efficiency and safe operation.

Towards addressing these challenges, this work presents a complete strategy for fixed-wing UAV path-tracking that relies on Model Predictive Control (MPC) [5-7] strategies combined with the nonlinear $\mathcal{L} 1$-navigation guidance law. The proposed approach respects the strict limitations on onboard computational resources, limited sensor suite and the need for accurate and safe navigation. Within this framework, MPC strategies are employed to achieve precise tracking of the inner-loops of the system and specifically pitch and bank angle control while respecting and satisfying the actuation limitations of the system and safety considerations encoded

This work was supported by the European projects ICARUS and SHERPA and the European Commision under the 7th Framework Programme.

All authors are with the Autonomous Systems Lab at ETH Zurich, Tannenstrasse 3, 8092 Zurich, Switzerland. email: konstantinos.alexis@mavt.ethz.ch as state constraints. Relying on recent advancements in the field, the proposed MPC is computed explicitly and therefore allows for seamless integration on the onboard avionics with very minimal requirements on processing power and memory. As the utilization of MPC shows its power only once a dynamics model of sufficient accuracy is available, grey-box system identification methods were employed as a preliminary step.

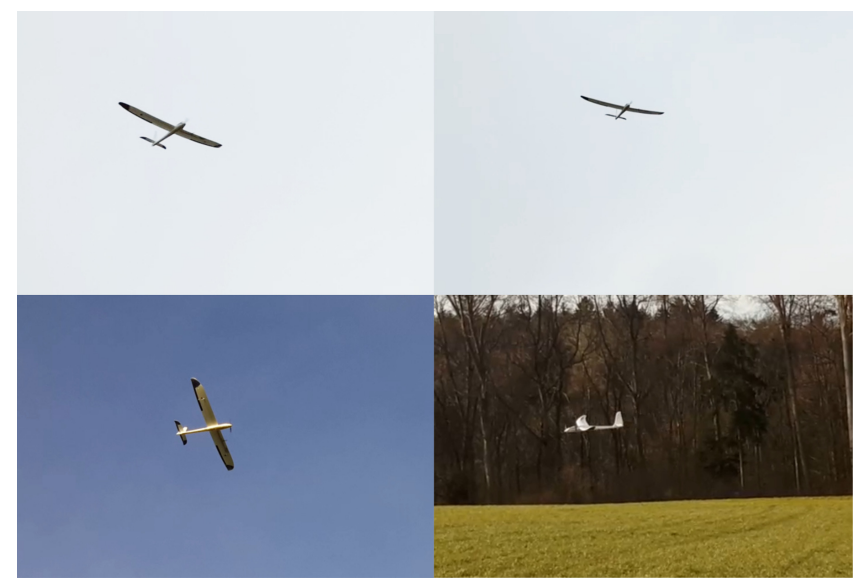

Fig. 1. Photos of the employed fixed-wing UAV experimental platform during flight operations.

The designed MPC ensures accurate steering of the UAV while respecting modeled state and input constraints. On top of this control augmentation, an $\mathcal{L}_{1}$ nonlinear navigation strategy [8] is deployed. This nonlinear guidance algorithm has shown to present superior performance in guiding UAVs along trajectories with sharply varying curvature [8]. All proposed control laws are implemented onboard the avionics of a small electric motorglider UAV, shown in Figure 1, along with all the state estimation and communication functions. Overall, a structed methodology to achieve precise reference tracking relying on tractable control laws and a very limited on-board sensory suite.

The remainder of this paper is structured as follows. In Section II the system overview is presented, followed by the description of the grey-box model and the identification methods in Section III. The employed fixed-wing UAV control strategy is detailed in Section IV, while evaluation flight results are presented in Section V. Finally, conclusions are drawn in the last Section VI. 


\section{SYSTEM OVERVIEW}

A brief overview of the characteristics of the experimental UAV along with those of the avionics and the properties of the onboard estimation algorithms is given below.

\section{A. Test Platform}

All experimental tests presented in this paper have been performed using the commercially available airframe shown in Figures 1 and 2. The platform features a conventional aileron, elevator and rudder control surface configuration and an electrically driven propulsion system using a frontmounted foldable fixed-pitch propeller. Its wingspan is $1.83 \mathrm{~m}$, the empty weight is $0.9 \mathrm{~kg}$ and the fully-equipped weight including a battery and all avionics s about $1.28 \mathrm{~kg}$.

The onboard avionics include multiple sensors in order to realize an efficient state estimation framework. Accelerations, angular rates, mangetometer and absolute air pressure readings are provided by a 10-axis ADIS16448 Inertial Measurement Unit (IMU). A u-Blox LEA-6H GPS receiver provides global position and aircraft velocities with respect to ground. In addition, an airspeed measuring system has been developed for precise measurement of low airspeeds common for small-scale UAVs. This system employs the Sensirion SDP600 sensor and exhibits an error of less than $5 \%$ at airspeeds $V_{T}=7 \mathrm{~m} / \mathrm{s}$, while measuring airspeeds up to ca. $28 \mathrm{~m} / \mathrm{s}(500 \mathrm{~Pa})$. Information of these sensors is fused as described in Section II-B to yield the estimated state of the aircraft.

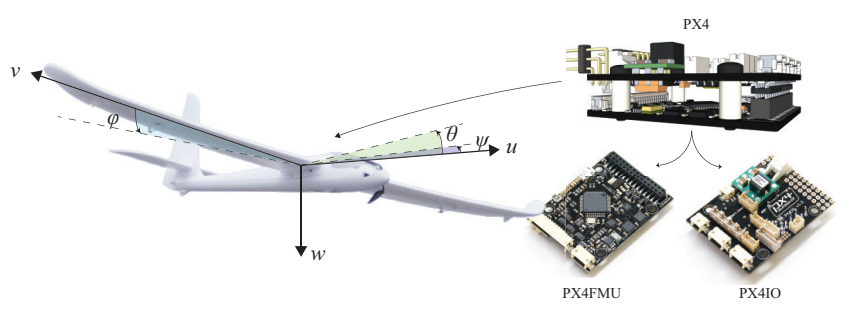

Fig. 2. The airframe (left) and the PX4 autopilot (right) used for development and flight testing.

The core of the onboard avionics is the Pixhawk PX4 [9] autopilot, an open-source and open-hardware project supported by ETH Zurich. The PX4 consists of the PX4FMU and the PX4IO boards as depicted in Figure 2. The PX4FMU is the main autopilot board. It features a $168 \mathrm{MHz}$ CortexM4F Microcontroller with 192KB SRAM and 1024KB Flash memory and runs the NUTTX real-time operating system. PX4IO serves as the low-level input/output board and provides failsafe RC-operations. Within the framework of this work, the baseline fixed-wing functionalities that are already part of the PX4 open software were replaced with the proposed model predictive controller along with improved state-estimation capabilities. The inner MPC-based roll- and pitch dynamics controllers are executed with a sampling period of $T_{s}^{M P C}=0.01 \mathrm{~s}$, while the $\mathcal{L}_{1}$-navigation loop is executed using $T_{s}^{\mathcal{L} 1}=0.05 \mathrm{~s}$. Logging of all state variables and inputs is performed every $T_{s}^{l o g}=0.025 \mathrm{~s}$.

\section{B. Onboard Estimation}

The control scheme outlined within this work, relies on the output of a custom on-board state-estimator, which utilizes the previously described set of sensors and its working principle is briefly summarized below. In essence, an indirect Extended Kalman Filter (EKF) uses acceleration and rotation rate measurements for propagation while employing static and dynamic pressure measurements as well as GPS and 3D magnetometer measurements as updates. The filter is an extended version of the authors' previous work [10] that offers robustness to GPS outages, estimates all three wind components, and can track motions with constant accelerations.

\section{FLIGHT DYNAMICS \& IDENTIFICATION}

The equations of motion for fixed-wing UAVs can be derived analytically from the Newton-Euler equations, with the main task being the determination of the external forces [11]. The UAV is subject to gravitational forces, propulsion forces and the forces resulting from aerodynamic phenomena. The resulting expressions are highly nonlinear and coupled. However, for non-aggressive maneuvering around level flight, linear models are valid and may be derived by assuming small perturbations from a given equilibrium. Such linear models typically decouple the longitudinal and lateral dynamics of the system and provide a framework for modelbased linear control synthesis. The linearized state equations for the longitudinal dynamics of small fixed-wing UAVs with a typical gliding configuration take the form [12]:

$$
\begin{aligned}
\mathbf{M}_{\text {lon }} \dot{\mathbf{x}}_{\text {lon }} & =\mathbf{A}_{\text {lon }}^{\prime} \mathbf{x}_{\text {lon }}+\mathbf{B}_{\text {lon }}^{\prime} u_{\text {elev }} \\
\mathbf{x}_{\text {lon }} & =\left[\begin{array}{lll}
u w q & \theta
\end{array}\right]^{T}
\end{aligned}
$$

where $u, w, q, \theta$ correspond to the body $x$-axis, $z$-axis velocities, the pitch rate and the pitch angle respectively, $u_{\text {elev }}$ corresponds to the elevator deflection and

$$
\begin{aligned}
\mathbf{M}_{\text {lon }}= & {\left[\begin{array}{cccc}
m & 0 & 0 & 0 \\
0 & m & 0 & 0 \\
0 & 0 & I_{y} & 0 \\
0 & 0 & 0 & 1
\end{array}\right], } \\
\mathbf{A}_{\text {lon }}^{\prime}= & {\left[\begin{array}{cccc}
X_{u} & X_{w} & X_{q}-m W_{e} & -m g \cos \theta_{e} \\
Z_{u} & Z_{w} & Z_{q}+m U_{e} & -m g \sin \theta_{e} \\
M_{u} & M_{w} & M_{q} & 0 \\
0 & 0 & 1 & 0
\end{array}\right] \mathbf{B}_{\text {lon }}^{\prime}=\left[\begin{array}{c}
X_{u_{e l e v}} \\
Z_{u_{\text {elev }}} \\
M_{u_{\text {elev }}} \\
0
\end{array}\right] }
\end{aligned}
$$

where $m$ is the mass, $I_{y}$ the inertia around the body $y-$ axis, $W_{e}, \theta_{e}$ are the trimming points of vertical velocity and pitch angle, and the elements of $\mathbf{M}_{\text {lon }}, \mathbf{A}_{\text {lon }}^{\prime}$ and $\mathbf{B}_{\text {lon }}^{\prime}$ form the stability and control derivatives of the UAV longitudinal dynamics.

Similarly, for the lateral dynamics the model takes the following form:

$$
\begin{aligned}
\mathbf{M}_{l a t} \dot{\mathbf{x}}_{l a t} & =\mathbf{A}_{l a t}^{\prime} \mathbf{x}_{l a t}+\mathbf{B}_{l a t}^{\prime} u_{\text {elev }} \\
\mathbf{x}_{l a t} & =\left[\begin{array}{lll}
v & p & r
\end{array}\right]^{T}
\end{aligned}
$$

where $v, p, r, \phi$ correspond to the body $y$-axis velocity, the roll and yaw rates and roll angle respectively, $u_{\text {ail }}$ is the aileron deflection, $u_{r u d}$ is the rudder deflection and 
TABLE I

$$
\begin{aligned}
\mathbf{M}_{\text {lat }} & =\left[\begin{array}{cccc}
m & 0 & 0 & 0 \\
0 & I_{x} & -I_{x z} & 0 \\
0 & -I_{x z} & I_{z} & 0 \\
0 & 0 & 0 & 1
\end{array}\right], \\
\mathbf{A}_{\text {lat }}^{\prime} & =\left[\begin{array}{cccc}
Y_{v} & Y_{p}+m W_{e} & Y_{r}-m U_{e} & m g \cos \theta_{e} \\
L_{v} & L_{p} & L_{r} & 0 \\
N_{v} & N_{p} & N_{r} & 0 \\
0 & 1 & \tan \theta_{e} & 0
\end{array}\right], \mathbf{B}_{\text {lat }}^{\prime}=\left[\begin{array}{cc}
Y_{u_{\text {ail }}} & Y_{u_{\text {rud }}} \\
L_{u_{\text {ail }}} & L_{u_{\text {rud }}} \\
N_{u_{\text {ail }}} & N_{u_{\text {rud }}} \\
0 & 0
\end{array}\right]
\end{aligned}
$$

where $I_{x}$ the inertia around the body $x$-axis, $I_{x z}$ the crossinertia term of the body $x, z$-axes and the elements of $\mathbf{M}_{\text {lat }}, \mathbf{A}_{\text {lat }}^{\prime}$ and $\mathbf{B}_{\text {lat }}^{\prime}$ form the stability and control derivatives of the UAV lateral dynamics. Some of these parameters may be accurately measured or estimated using CAD tools and simple experiments. However, especially the control and stability derivatives relevant with aerodynamic effects are typically hard to be estimated using only first-principles approaches. Therefore, the aforementioned models are employed as grey-box system structures within a system identification framework that uses real flight-data to estimate the unknown or roughly estimated parameters.

Towards high fidelity system identification, a structured way of defining excitation signals, evaluating the quality of recorded data, conducting frequency-domain identification steps and evaluating the estimated models is employed. All three $u_{\text {elev }}, u_{\text {ail }}, u_{\text {rud }}$ inputs that excite the vehicle longitudinal dynamics are manipulated using chirp signals that cover a wide spectrum area expected to contain the UAV dominant dynamics. Subsequently, the quality of the recorded flight data is evaluated in the frequency-domain by checking the coherence between the vehicle states and the corresponding dominant inputs: data of high quality that are useful for linear system identification are available as long as the input $u_{i}$ to output $y_{j}$ coherence $\gamma_{u_{i}, y_{j}}$ is sufficiently high and typically above $\gamma_{u_{i}, y_{j}} \geq 0.6$.

Once the stage of flight data recording and data preparation is completed, the frequency response of the data is computed using the Fast Fourier Transform (FFT). The solution of the MIMO identification problem involves determining the model matrices $\mathbf{A}_{l o n}^{\prime}, \mathbf{A}_{l a t}^{\prime}, \mathbf{M}_{l o n}, \mathbf{M}_{l a t}, \mathbf{B}_{\text {lon }}^{\prime}, \mathbf{B}_{\text {lat }}^{\prime}$ that produce a frequency-response matrix $\hat{\mathbf{T}}_{c}$ that most closely matches the frequency responses $\mathbf{T}$ obtained from the experimental results. The optimization algorithm provides the capability to weight the frequency response in a way that the subset that is mostly excited from the inputs plays a more important role. This prevents errors caused by overfocusing in nonlinearities outside the main flight envelope. The weighted cost function to be minimized takes the form [13]:

$$
\begin{aligned}
\mathbf{J} & =\sum_{l=1}^{n_{T F}} \sum_{\omega_{1}}^{\omega_{n} \omega} W_{\gamma}\left(\omega_{i}\right)\left[W_{g}\left(\left|\hat{\mathbf{T}}_{c}\left(\omega_{i}\right)\right|-\left|\mathbf{T}\left(\omega_{i}\right)\right|\right)^{2}+W_{p}\left(\left\langle\hat{\mathbf{T}}\left(\omega_{i}\right)_{c}-\angle \mathbf{T}\left(\omega_{i}\right)\right)^{2}\right],\right. \\
W_{\gamma}(\omega) & =\left[1.58\left(1-\exp ^{-\gamma_{u_{i}}^{2}, y_{j}}\right)\right]^{2}, \quad W_{g}=1.0, \quad W_{p}=0.01745,
\end{aligned}
$$

where $W_{\gamma}\left(\omega_{i}\right)$ is the weighting function that depends on the input-output coherence at frequency $\omega_{i}, n_{\omega}$ is the number of frequency points, $\omega_{1}$ and $\omega_{n_{\omega}}$ are the lowest and highest frequencies of the fit, $n_{T F}$ is the number of considered input/output relations of the MIMO system and $\gamma_{u_{i}, y_{i}}$ indicates the coherence between input $u_{i}$ and output $y_{j}$. Employing
ESTIMATED MODES OF THE LONGITUDINAL DYNAMICS

\begin{tabular}{|lll|}
\hline Mode & Natural Frequency (rad/s) & Damping Ratio \\
\hline Phugoid & 0.799 & 0.653 \\
Short-Period Pole 1 & 10.3 & - \\
Short-Period Pole 2 & 10.8 & - \\
\hline
\end{tabular}

these identification methods, sufficiently precise lateral and longitudinal models are derived as shown in Figures 3 and 4. The trim point was around level flight and specifically $U_{e}=$ $8.6 \mathrm{~m} / \mathrm{s}, W_{e}=0.6 \mathrm{~m} / \mathrm{s}, \theta_{e}=0.045 \mathrm{rad}, v_{e}=0.0$ (lateral velocity trim point) $p_{e}=q_{e}=r_{e}=0$ (attitude rates trim point). Note that the models are validated against data not used during the system identification process.
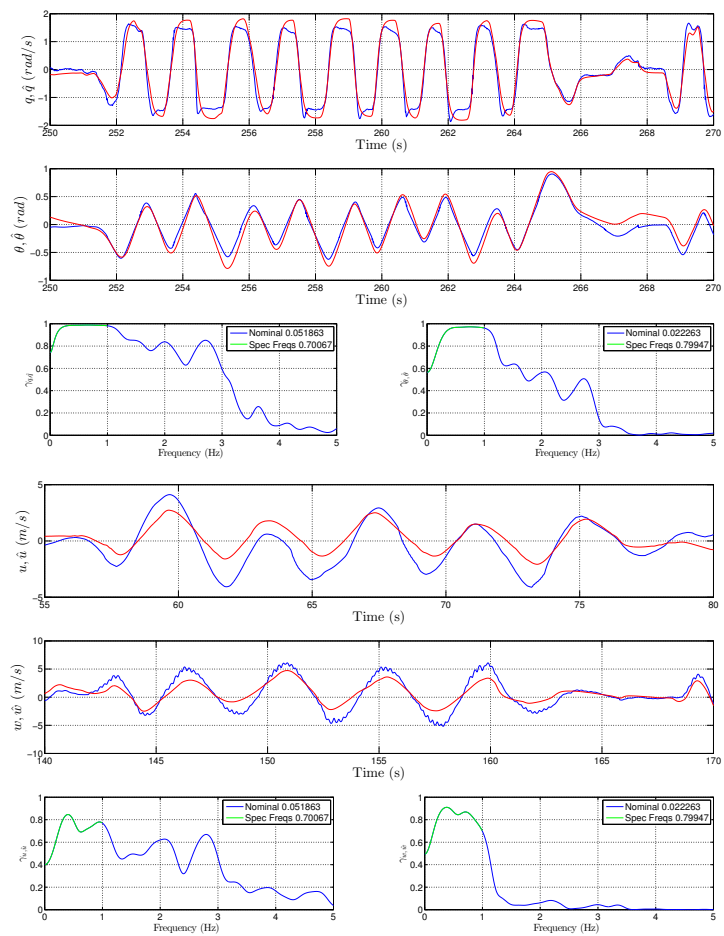

Fig. 3. Longitudinal dynamics identification fidelity validation. As shown the pitch rate $q$ and pitch angle $\theta$ are precisely identified while for the velocity components $u, w$ sufficient fidelity is achieved.

Apart from the fit of the estimated responses against the experimentally recorded data, a model used for control also owes to be physically consistent. This may be qualitatively evaluated based on the model eigenvalues. Tables I and II present the dominant pole time constants and damping ratios for the longitudinal and lateral dynamics respectively. $\mathrm{n}$ summary, sufficiently accurate and physically consistent models were derived for the longitudinal and lateral dynamics of the employed UAV. These models set the basis for model-based control synthesis.

\section{CONTROL STRATEGY}

The aforementioned identified system dynamics enable the utilization of model-based control synthesis towards a structured and formal way of achieving optimal responses 

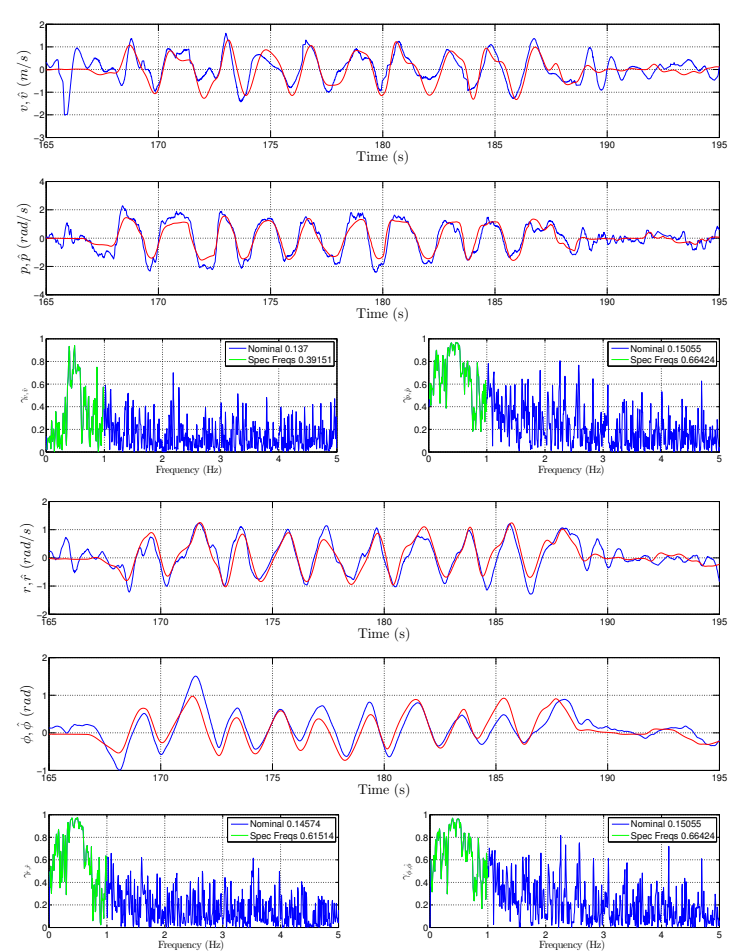

Fig. 4. Lateral dynamics identification fidelity validation. As shown the attitude rates $p, r$ and the roll angle are accurately captured while the lateral velocity $v$ component also presents a sufficient fit.

TABLE II

ESTIMATED MODES OF THE LATERAL DYNAMICS

\begin{tabular}{|lll|}
\hline Mode & Natural Frequency $(\mathrm{rad} / \mathrm{s})$ & Damping Ratio \\
\hline Spiral & 0.76 & - \\
Dutch Roll & 6.68 & 0.259 \\
Roll & 11.7 & - \\
\hline
\end{tabular}

that respect the actuation limitations of the UAV and ensure imposed state constraints encoding a safe flight envelope. Within this work, receding horizon control strategies for the roll and pitch dynamics are combined with a nonlinear guidance law to form a complete efficient path-tracking scheme.

\section{A. Roll/Pitch MPC Loops}

The UAV inner loops controllers, namely those responsible for roll and pitch tracking and thereby also handling the angle of attack safety constraints are particularly important. Therefore, this work goes beyond the typical methods that mostly rely on cascaded PID loops [11] and employs an advanced model predictive control framework. Correspondingly, two MPCs are computed namely for the longitudinal and lateral UAV dynamics.

1) State and Input Constraints: One of the particularly special properties of MPC that make it attractive for flying vehicles is its inherent capability to account for state and input constraints. Using input and state constraints, a safe subset of the flight envelope may be defined. The following constraints were specifically encoded within the framework of this work:

$$
\begin{aligned}
& {\left[\begin{array}{cc}
\mathbf{I}_{4 \times 4} & \mathbf{0}_{4 \times 4} \\
\mathbf{0}_{4 \times 4} & -\mathbf{I}_{4 \times 4}
\end{array}\right]\left[\begin{array}{c}
q \\
\theta \\
\alpha \\
u_{\text {elev }} \\
q \\
\theta \\
\alpha \\
u_{\text {elev }}
\end{array}\right] \leq\left[\begin{array}{c}
5 \mathrm{rad} / \mathrm{s} \\
\pi / 2 \mathrm{rad} \\
\pi / 8 \mathrm{rad} \\
\pi / 16 \\
5 \mathrm{rad} / \mathrm{s} \\
\pi / 2 \mathrm{rad} \\
\infty \\
\pi / 16
\end{array}\right]} \\
& {\left[\begin{array}{cc}
\mathbf{I}_{3 \times 3} & \mathbf{0}_{3 \times 3} \\
\mathbf{0}_{3 \times 3} & -\mathbf{I}_{3 \times 3}
\end{array}\right]\left[\begin{array}{c}
p \\
\phi \\
u_{\text {ail }} \\
p \\
\phi \\
u_{\text {ail }}
\end{array}\right] \leq\left[\begin{array}{c}
5 \mathrm{rad} / \mathrm{s} \\
\pi / 2 \mathrm{rad} \\
\pi \\
5 \mathrm{rad} / \mathrm{s} \\
\pi / 2 \mathrm{rad} \\
\pi / 16
\end{array}\right]}
\end{aligned}
$$

The constraint on the angle of attack is not directly introduced, as the employed longitudinal dynamics model does not contain $\alpha$ directly as a state. However, as $\alpha=$ $\arctan (w / u)$ this constraint is introduced as a polyhedric constraint expressed in terms of the two velocities $u, w$. Figure 5 illustrates the values that the angle of attack takes for perturbations of the vehicle body velocities around the trim point $U_{e}, W_{e}$. Applying the constraint on $\alpha \geq p i / 8$ defines a subset of this set of values that has to be avoided. The line that separates the "safe" and unsafe "area" is defined by $w / u=\tan (\pi / 8)$. This polyhedric constraint is then introduced into the convex optimization problem based on the recent advancements in the field [14].

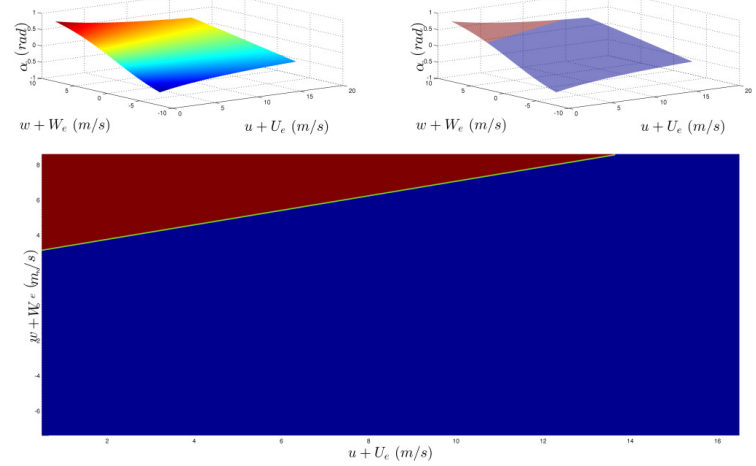

Fig. 5. Implementation of the angle of attack constraint as a polyhedric constraint on the body velocities of the UAV. The upper left plot illustrates the values of the angle of attack for different perturbations of the $u, w$ velocities, while the top right plot presents with red color the cases that $\alpha \geq p i / 8$. The plot on the bottom presents this constraint in $2 \mathrm{D}$ regarding the velocities of the vehicle.

2) MPC Computation: Provided the discretized representations of the vehicle dynamics given in (1),(3) as well as the constraints (6),(7) two predictive controllers are computed in a multiparametric fashion [15-19]. Using a quadratic norm as a metric of optimality, the predictive controller, for a prediction horizon $N$, consists of computing the optimal control sequence $\mathbf{U}_{\xi}^{\mathbf{N}}=\left[u_{\xi}(0), \ldots, u_{\xi}(N-1)\right]$ that minimizes the following objective:

$$
\begin{aligned}
\mathbf{J}\left(y_{\xi, 0}, U_{\xi}^{N}\right)= & \min _{U_{\xi}^{N}}\left\{\sum_{k=0}^{N-1} \mathbf{y}_{\xi, k}^{T} \mathbf{Q}_{M \times M} \mathbf{y}_{\xi, k}+\mathbf{u}_{\xi, k}^{T} \mathbf{R}_{L \times L} \mathbf{u}_{\xi, k}\right\} \\
\text { s.t. } & \text { equation (1),(3),[(6)/(7)] }
\end{aligned}
$$


where $\xi \rightarrow$ lon, lat, $\mathbf{Q}_{M \times M} \succeq \mathbf{0}, \mathbf{R}_{L \times L} \succeq \mathbf{0}$ are the weighting matrices of the outputs and the manipulated variables respectively. Note that for the case of lateral dynamics, the output vector is considered to be the roll angle $\phi$ and roll rate $p$, while for the case of longitudinal dynamics the output vector is considered to be the pitch angle $\theta$ and the pitch rate $q$ :

$$
\begin{aligned}
\mathbf{y}_{\text {lon }} & =\mathbf{C}_{\text {lon }} \mathbf{x}_{\text {lon }}, \mathbf{y}_{\text {lon }}=\left[\begin{array}{ll}
q & \theta
\end{array}\right]^{T} \\
\mathbf{y}_{\text {lat }} & =\mathbf{C}_{\text {lat }} \mathbf{x}_{\text {lat }}, \mathbf{y}_{\text {lat }}=\left[\begin{array}{ll}
p
\end{array}\right]^{T} \\
\mathbf{C}_{\text {lon }} & =\left[\begin{array}{llll}
0 & 0 & 1 & 0 \\
0 & 0 & 0 & 1
\end{array}\right], \mathbf{C}_{\text {lat }}=\left[\begin{array}{llll}
0 & 1 & 0 & 0 \\
0 & 0 & 0 & 1
\end{array}\right]
\end{aligned}
$$

The remaining of the system states are used to predict the system dynamics but not used for reference tracking.

3) Explicit MPC Implementation: Although a straightforward implementation of such a model predictive control strategy relies on solving a convex optimization problem online, the fact that the available onboard processing power is limited motivated following a different approach. As proven in the recent years, a special property of such a receding horizon strategy is the fact that it can be translated to an equivalent explicit representation [20]. The control action then takes then a piecewise affine form:

$$
\mathbf{u}_{\xi}(k)=\mathbf{F}_{\xi}^{r} \mathbf{y}_{\xi}(k)+\mathbf{G}_{\xi}^{r}, \text { if } \mathbf{y}_{\xi}(k) \in \Pi_{\xi}^{r}
$$

where $\Pi_{\xi}^{i}, \xi \rightarrow$ lon, lat $, r=1, \ldots, N_{\xi}^{r}$ are the regions of the receding horizon control strategy. The $r$-th control law is valid if the output vector $\mathbf{y}_{\xi}(k)$ is contained in a convex polyhedral region $\Pi_{\xi}^{r}=\left\{\mathbf{y}_{\xi}(k) \mid \mathbf{H}_{\xi}^{r} \mathbf{y}_{\xi}(k) \leq \mathbf{K}_{\xi}^{r}\right\}$ computed and described in $h$-representation during the explicit controller derivation [21]. The controller is equivalently translated to a mapping between feedback gains and affine terms $\mathbf{F}_{\xi}^{r}, \mathbf{G}_{\xi}^{r}$ and corresponding polyhedric regions $\Pi_{\xi}^{r}$. This explicit controller is equivalent to its online counterpart in the sense that for identical state trajectories, both produce the same control actions, and therefore share the same stabilizing and optimality properties [22]. This fact enables the seamless real-time execution of this controller. In the framework of this work, the real time code is described in Algorithm 1 and corresponds to an extension of the table traversal algorithm $[18,21]$ that also supports multiple inputs.

\section{B. Cascaded $\mathcal{L}_{1}-$ Navigation and Altitude Control}

The $\mathcal{L}_{1}$-navigation logic [8] introduces an adaptive way of selecting a reference point on the global desired trajectory, and consequently generates a lateral acceleration command using that reference point. With the corresponding symbols noted in Figure 6, the reference point is select on the desired path at a tunable distance $L_{1}$ ahead of the vehicle and then the lateral acceleration command is given by the following expression:

$$
\alpha_{s_{r e f}}=2 \frac{V_{T}^{2}}{L_{1}} \sin \eta
$$

where $\eta$ denotes the angle between the velocity vector of the vehicle and the vector created from the UAV center of gravity

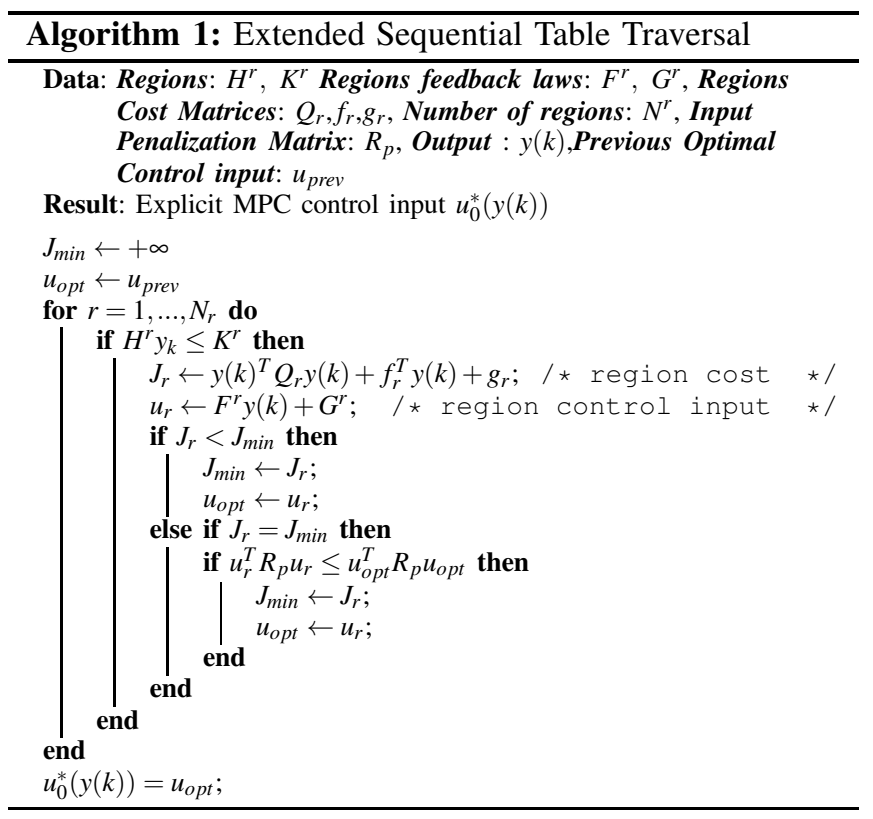

and the selected reference point according to the $L_{1}$ distance. As intuitively understood, the direction of the acceleration command depends on the sign of $\eta$ which essentially means that the UAV will tend to align its velocity direction with that of the $L_{1}$ line segment. Furthermore, as shown in Figure 6, a circular path can always be defined by the position of the vehicle and the vector tangential to the vehicle velocity. The reference acceleration is then equal to the centripetal acceleration needed to track this instantaneous circular path generated at every point in time. This fact may be verified by setting $L_{1}=2 R \sin \eta$ which consequently yiels to $\alpha_{s_{r e f}}=$ $2 \frac{V_{T}^{2}}{L_{1}} \sin \eta \Rightarrow \alpha_{s_{r e f}}=\frac{V_{T}^{2}}{R}=$ centripetal acceleration. Therefore, the $\mathcal{L}_{1}$ nonlinear guidance law will always produce a lateral acceleration that will enable tracking of a circle of any radius $R$. This is naturally subject to the assumption that the lower-level loops of the system are able to follow such a command. Therefore, efficient tuning of the $\mathcal{L}_{1}$-navigation strategy becomes possible only once the attitude loops are properly tuned. Once this is achieved, the acceleration command is translated to a banking angle reference according to a linear mapping for different airspeeds of the system $\phi^{r}=f\left(\alpha_{s_{r e f}}\right)$ which can be kinematically derived. Thorough analysis of the performance and robustness properties of the $\mathcal{L}_{1}$ guidance may be found in [8]. It is noted that the utilized implementation of the $\mathcal{L}_{1}$ guidance is based on the PX4 open-source project [9].

Along with this implementation of the $\mathcal{L}_{1}$-navigation, a slew-rate controlled proportional altitude controller which provides references to the pitch MPC is implemented. The pitch reference control action takes the following form:

$$
\theta^{r}=K_{P}^{z} \sigma\left(z^{r}-z\right)
$$

where $z^{r}$ corresponds to the altitude reference, $z$ is the current estimated altitude of the UAV, $\sigma(\cdot)$ corresponds to the slew- 


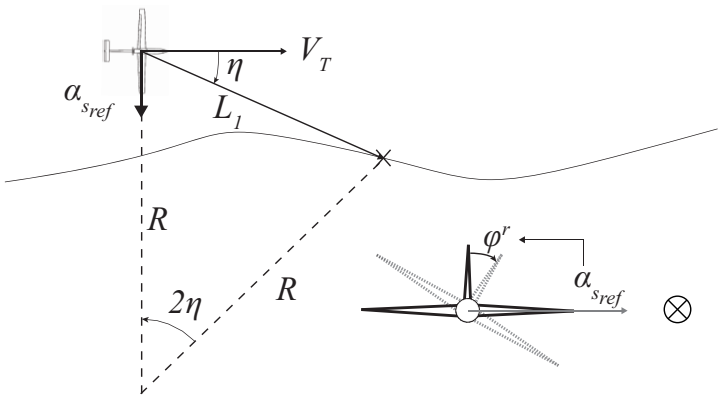

Fig. 6. $\mathcal{L}_{1}$-navigation based selection of adapting reference point and corresponding acceleration/banking angle reference command.

TABLE III

COMPUTATIONAL DEMANDS OF THE IMPLEMENTED EXPLICIT MPC.

\begin{tabular}{|llll|}
\hline Application(s) & CPU Load & RAM & Flash memory \\
\hline R/P (roll/pitch) MPC & $3.6 \%$ & $3.78 \mathrm{~KB}$ & $42.45 \mathrm{~KB}$ \\
R/P MPC $+\mathcal{L}_{1}$ Navigation & $4.4 \%$ & $4.04 \mathrm{~KB}$ & $47.05 \mathrm{~KB}$ \\
R/P PID Control & $0.7 \%$ & $3.78 \mathrm{~KB}$ & $26.85 \mathrm{~KB}$ \\
State Estimation & $18.3 \%$ & $6.1 \mathrm{~KB}$ & $159.6 \mathrm{~KB}$ \\
All other & $39.2 \%$ & $118.2 \mathrm{~KB}$ & $844.5 \mathrm{~KB}$ \\
\hline
\end{tabular}

rate function and $K_{P}^{z}$ is the tunable proportional gain.

\section{FLIGHT RESULTS}

The proposed control framework was implemented on the onboard avionics taking into account its strict limitations on memory as well as processing power. Appropriate selection of the prediction horizon is fundamental since it has a significant influence on the controller performance, it depends on the fidelity of the available model while at each increment leads to considerably larger explicit MPC representations and therefore becomes more memory demanding. Focusing on the fast modes of the longitudinal and lateral dynamics as shown in Tables I and II, the prediction horizons were set to $N=6$ and $N=5$ respectively, a value that leads to explicit representations realizable on-board. To provide further insight on how lightweight the computational requirements of the proposed control framework are, Table III summarizes the computational demands of the dominant functions running on board as well as the corresponding numbers in case of a PID controller for the attitude dynamics. Herein, the numbers given include CPU- and memory consumption due to housekeeping tasks such as data logging. As expected, the implemented MPC-scheme is computationally more demanding than a standard PID scheme. However, due to its explicit character, the overall computation demands, even for the MPC $+\mathcal{L}_{1}$-navigation scheme, are still very low: Less than $5 \%$ of CPU load and about $4 \mathrm{~KB}$ of RAM is taken by the tuned versions of the roll and pitch MPCs. The low computational demands are especially obvious when comparing the MPC algorithm to other onboard tasks such as the state estimator or other housekeeping applications.

The penalization matrices of the predictive controllers were tuned with soft penalization on the attitude rates, significant penalization of the angle and a penalization of the input signal that leads to smooth control actions. Figures 7 and 8 illustrate the results of roll and pitch tracking respectively. Two sets of closed-loop responses are depicted, namely for larger and smaller penalization of the roll and pitch angles, while the remaining parameters were identical. Note that within these and all the other presented responses, the throttle is set to a fixed value that in trim conditions leads to a longitudinal velocity of approximately $9 \mathrm{~m} / \mathrm{s}$.
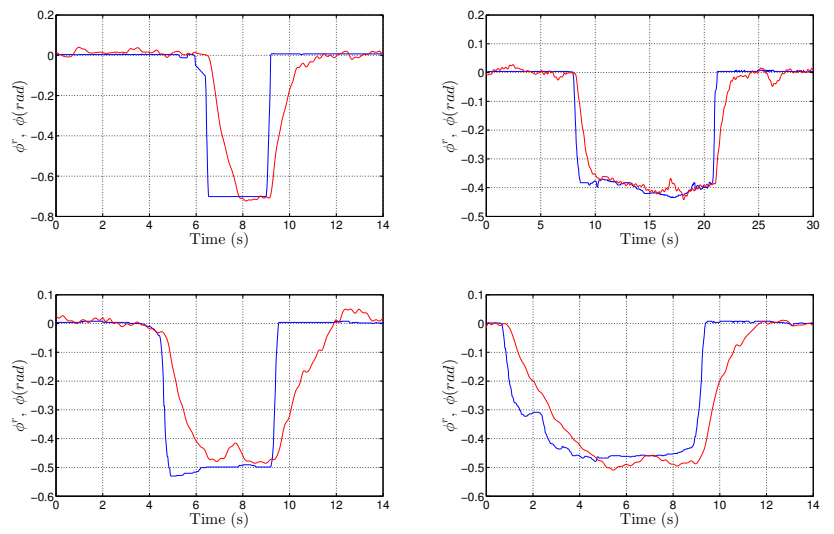

Fig. 7. Results of roll reference tracking for two different MPC tuning cases, namely a more aggressive (upper responses) and one with less penalization on the $\mathbf{Q}$ matrix (bottom responses). Blue color is used for the references and red for the recorded responses.
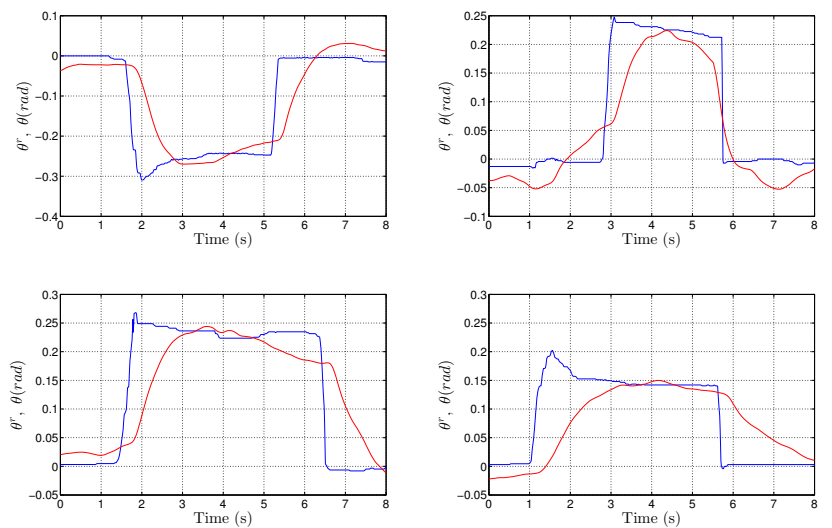

Fig. 8. Results of pitch reference tracking for two different MPC tuning cases, namely a more aggressive (upper responses) and one with less penalization on the $\mathbf{Q}$ matrix (bottom responses). Blue color is used for the references and red for the recorded responses.

Favoring smoother responses, the MPCs leading to the responses of the bottom part of the previously presented figures were selected. Once satisfactory closed-loop roll and pitch responses are ensured while also accounting for safety considerations related with stall effects, the $\mathcal{L}_{1}$-navigation strategy parameters may be tuned. The direction of tuning was based on the desire to have a sharp response which however does not command the vehicle to bank more than 30 degrees mostly for safety reasons and the desire to execute such path-tracking tasks at very low speeds. An indicative result is shown in Figure 9 where the achieved path is compared against the reference.

Consequently, the path tracking capabilities were tested in 


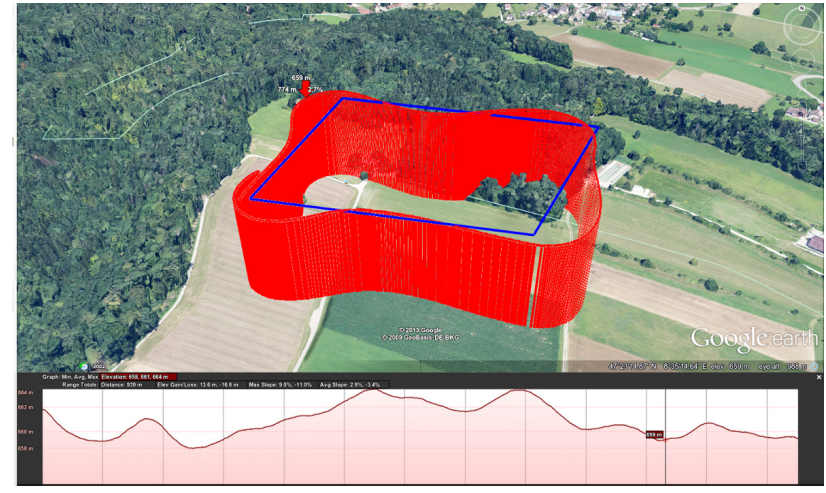

Fig. 9. Path-tracking response of the proposed combined $\mathrm{MPC} / \mathcal{L}_{1-}$ navigation strategy. As shown ,accurate responses are achieved for a sharp rectangle path. The wind conditions were $8 \mathrm{~km} / \mathrm{h}$ average wind with up to $18 \mathrm{~km} / \mathrm{h}$ wind gusts.

the case of a circular loitering reference which is a common reference path during surveillance or communication-relay operations. Figure 10 present the achieved results which are sufficiently accurate despite the relatively windy conditions, the small size of the aircraft and the short radius of the circle.

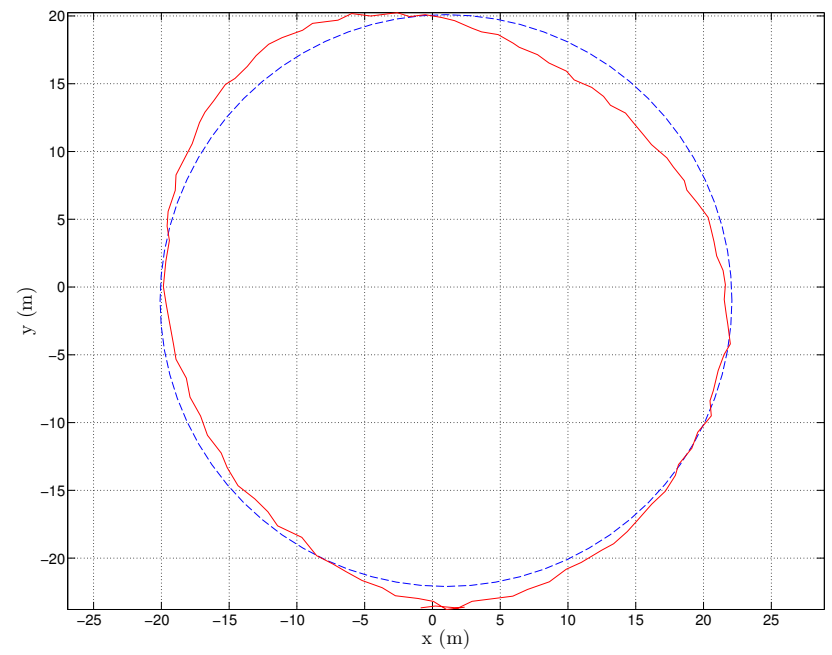

Fig. 10. Circular-path tracking of the combined MPC and $\mathcal{L}_{1}$ control law.

Overall, the experimental studies indicate the efficiency of the proposed combination of the nonlinear $\mathcal{L}_{1}$-guidance with the newly introduced explicit model predictive control law for the inner-loops of such a small fixed-wing UAV.

\section{CONCLUSIONS}

A new control approach for the guidance of a small UAV that relies on the combination of MPC approaches responsible for the inner-loop stabilization and feedback control, along with the nonlinear $\mathcal{L}_{1}$-navigation strategy that is responsible for providing steering commands that can lead to precise path tracking is presented. The MPC strategies provide optimal responses while respecting the system input and state constraints. Being explicitly computed, it allows for computationally lightweight and seamless integration on the onboard avionics. As MPC provides accurate steering, tuning and safe manipulation of the vehicle actuators, tuning of the $\mathcal{L}_{1}$-guidance towards precise waypoint navigation is achieved. A set of experiments indicate the achieved performance on all the levels of the vehicle output tracking.

\section{REFERENCES}

[1] P. Rudol and P. Doherty, "Human body detection and geolocalization for uav search and rescue missions using color and thermal imagery," in Aerospace Conference, 2008 IEEE, 2008, pp. 1-8.

[2] E. R. Hunt, W. D. Hively, S. J. Fujikawa, D. S. Linden, C. S. T. Daughtry, and G. W. McCarty, "Acquisition of nir-green-blue digital photographs from unmanned aircraft for crop monitoring," Remote Sensing, vol. 2, no. 1, pp. 290-305, 2010.

[3] M. Burri, J. Nikolic, C. Hurzeler, G. Caprari, and R. Siegwart, "Aerial service robots for visual inspection of thermal power plant boiler systems," in Applied Robotics for the Power Industry (CARPI), 2012 2nd International Conference on, 2012, pp. 70-75.

[4] F. Nex and F. Remondino, "Uav for 3d mapping applications: A review," Applied Geomatics, pp. 1-15, 2013.

[5] E. F. Camacho and C. Bordons, Model Predictive Control. Springer, 2003

[6] K. Alexis, G. Nikolakopoulos, and A. Tzes, "Model predictive quadrotor control: attitude, altitude and position experimental studies," Control Theory \& Applications, IET, vol. 6, no. 12, pp. 1812-1827, 2012

[7] C. Papachristos, K. Alexis, and A. Tzes, "Model predictive hoveringtranslation control of an unmanned tri-tiltrotor," in 2013 International Conference on Robotics and Automation, Karlsruhe, Germany, 2013, pp. 5405-5412.

[8] S. Park, J. Deyst, and J. P. How, "A new nonlinear guidance logic for trajectory tracking," in AIAA Guidance, Navigation, and Control Conference and Exhibit, 2004, pp. 16-19.

[9] PIXHAWK Autopilot Research Project, "https://pixhawk.ethz.ch/."

[10] S. Leutenegger and R. Siegwart, "A low-cost and fail-safe inertial navigation system for airplanes," in Robotics and Automation (ICRA), 2012 IEEE International Conference on, May 2012, pp. 612-618.

[11] Brian L. Stevens and Frank L. Lewis, Aircraft Control and Simulation. Wiley Interscience, 1992.

[12] A. Dorobantu, A. Murch, B. Mettler, and G. Balas, "Frequency domain system identification for a small, low-cost, fixed-wing uav," in Proceedings of the 2011 AIAA Guidance, Navigation, and Control Conference, 2011.

[13] Mark B. Tischler, Robert K. Remple, Aircraft and Rotorcraft System Identification: Engineering methods with Flight-Test examples. American Institute of Aeronautics and Astronautics (AIAA).

[14] J. Loefberg, "Yalmip : A toolbox for modeling and optimization in MATLAB," in Proceedings of the CACSD Conference, Taipei, Taiwan, 2004. [Online]. Available: http://users.isy.liu.se/johanl/yalmip

[15] M. Herceg, M. Kvasnica, C. Jones, and M. Morari, "Multi-Parametric Toolbox 3.0," in Proc. of the European Control Conference, Zürich, Switzerland, July 17-19 2013, pp. 502-510.

[16] M. Baotic, "Optimal control of piecewise affine systems," Ph.D. dissertation, Swiss Federal Institute of Technology Zurich, 2005.

[17] K. Alexis, C. Huerzeler, and R. Siegwart, "Hybrid modeling and control of a coaxial unmanned rotorcraft interacting with its environment through contact," in 2013 International Conference on Robotics and Automation (ICRA), Karlsruhe, Germany, 2013, pp. 5397-5404.

[18] G. Darivianakis, K. Alexis, M. Burri, and R. Siegwart, "Hybrid predictive control for aerial robotic physical interaction towards inspection operations," in 2014 International Conference on Robotics and Automation (ICRA), Hong Kong, China, 2014, accepted.

[19] K. Alexis, G. Nikolakopoulos, and A. Tzes, "Constrained-control of a quadrotor helicopter for trajectory tracking under wind-gust disturbances," in MELECON 2010-2010 15th IEEE Mediterranean Electrotechnical Conference. IEEE, 2010, pp. 1411-1416.

[20] M. Kvasnica, Real-Time Model Predictive Control via MultiParametric Programming: Theory and Tools. VDM Verlag, 2009.

[21] M. Kvasnica, I. Rauova, and M. Fikar, "Automatic code generation for real-time implementation of model predictive control," in ComputerAided Control System Design (CACSD), 2010 IEEE International Symposium on, sept. 2010, pp. $993-998$.

[22] A. Bemporad, "Modeling, control, and reachability analysis of discrete-time hybrid systems," University of Sienna, March 2003. 